\title{
Study for the Safety of Ships' Nonlinear Rolling Motion in Beam Seas
}

\author{
Zhan-Jun Long** Seung-Keon Lee ${ }^{\dagger} \cdot J a e^{-H u n}$ Jeong** $\cdot$ Sung-Jong Lee*** \\ † Professor, Dept. of Naval Architecture and Ocean Engineering, Pusan National University, Pusan 609-735, Korea \\ *,**,*** Dept. of Naval Architecture and Ocean Engineering, Pusan National University, Pusan 609-735, Korea
}

\begin{abstract}
Vessels stability problems need to resolve the nonlinear mathematical models of rolling motion. For nonlinear systems subjected to random excitations, there are very few special cases can obtain the exact solutions. In this paper, the specific differential equations of rolling motion for intact ship considering the restoring and damping moment have researched firstly. Then the partial stochastic linearization method is applied to study the response statistics of nonlinear ship rolling motion in beam seas. The ship rolling nonlinear stochastic differential equation is then solved approximately by keeping the equivalent damping coefficient as a parameter and nonlinear response of the ship is determined in the frequency domain by a linear analysis method finally.
\end{abstract}

Key words : Ship stability, Non-linear dynamical systems, Roll damping, Safety of ships

\section{Introduction}

For the rolling motion of ships, there are many authors who have proposed different models for damping and restoring moment in some assumptions. The response of rolling motions of a ship can be adequately described by a linear equation if the rolling angle is small. However, as the amplitude of oscillation increases, nonlinear effects come into play(Lee, et al., 2007, Surendran, et al., 2005, Surendran, et al., 2007). Then vessels stability problems need to resolve the nonlinear mathematical models of rolling motion. But there are very few special cases which can obtain the exact solutions for nonlinear systems subjected to random excitations. For this reason, several approximate techniques have been developed for the probability density function of nonlinear systems. Generally, these methods can be subdivided in two groups(Brukner and Lin, 1987, Cai and Lin, 1988, Caughey, 1986, Langley, 1988, Polidori and Beck, 1996): quasi linear methods and quite nonlinear methods. Quasi linear methods substitute the solution of a nonlinear problem by a sequence of related linear ones and one of the most common techniques in this group is the stochastic linearization method(Proppe, et al., 2003, Socha, 2005). Quite nonlinear methods substitute the solution of a given nonlinear problem by a nonlinear know one(Belenky, et al., 1998). Recently, an alternative technique called stochastic nonlinearization has been suggested, in which one replaces the original nonlinear stochastic differential equation by another 'close' nonlinear equation, possessing an exact solution(Zhu, et al., 1994).

Recently, there are many scholars who are interesting in the nonlinear rolling oscillation because of the safety of ship received more and more recognition by the government or international maritime organizations. A great deal of methods are fully developed at the same period excited by the foundation. An approximation method for the conventional linear and linear plus quadratic rolling oscillation was presented in Ref.(Dalzell, 1978). Concentrated on the phenomena of ship capsizing due to an initial inclination, nonlinear governing equation of roll was linearized and converted into a Mathew-type equation and the stable, critical and unstable conditions for ship stability has suggested in Ref. (Lee, 2000). An experimental to nonlinear rolling motion of a ship model in regular wave have tested in Ref.(Contento, et al., 1996). An exact solution for free nonlinear uncoupled rolling or pitching motion of a submerged vehicle has proposed in Ref.(Birman, 1986). Various types of damping associated with rolling and empirical relationships for roll damping have been presented in Ref.(Chakrabarti, 2001). Surendran et al. discussed the linear response of a $\mathrm{Ro}^{-}$- $\mathrm{Ro}$ ship and considered various types of damping and restoring moments to show the nonlinear response in regular waves(Surendran and Venkata Ramana Reddy, 2003).

\footnotetext{
† Corresponding author: Seung Keon Lee, Leesk@pusan.ac.kr 051)510-2411

* Long@pusan.ac.kr, 051)510-2755

** wave0120@pusan.ac.kr 051)510-2755

***kafa789@daum.net 051)510-2755
} 
In this study, the stochastic linearization and nonlinearization techniques are combined, which is specially designed for rolling motion systems with both nonlinear damping and nonlinear restoring force. Instead of the classical stochastic linearization technique, where both nonlinear damping and nonlinear restoring force are replaced by their respective linear counterparts, here we use only a partial linearization. The equivalent damping parameter is obtained by solving a nonlinear algebraic equation either analytically or numerically. The proposed procedure yields simple equations to determine the desired probabilistic characteristics.

\section{Concept of partial linearization for nonlinear stochastic system}

Partial linearization method is applicable for the system with stochastic differential equations. We will present a single second order equation here and consider the basic nonlinear system as the following equation(Epele, et al., 1985, Haddara and Zhang, 1994, Ochi, 1986)

$$
\ddot{Y}+f(Y, \dot{Y})+g(Y)=M(t)
$$

Where $\mathrm{f}$ and $\mathrm{g}$ are known nonlinear functions, the excitation $\mathrm{M}(\mathrm{t})$ is assumed to be a Gaussian white noise with spectral density $K$. Then, the nonlinear damping force $f$ can be replace by an equivalent linear one,

$$
\ddot{Y}+\beta_{e} \dot{Y}+g(Y)=M(t)
$$

In order to obtain the linear damping coefficient of the substituting system(2), the key issue is to insure the equivalent damping coefficient $\beta e$ has the same effect as the function $\mathrm{f}$ in stochastic system(6). The criterion for selecting $\beta e$ is that the average energy dissipation remains the same,

$$
E[\dot{Y} \cdot f(Y, \dot{Y})]=\beta_{e} E\left[\dot{Y}^{2}\right]
$$

Which represent the average work per unit time performed by the original nonlinear damping force and the equivalent linear damping force.

$$
E[\dot{Y} \cdot f(Y, \dot{Y})]-\beta_{e} E\left[\dot{Y}^{2}\right]=\min
$$

Also, the stochastic linearization uses the least mean square criterion as follows

$$
E\left\{\left[f(Y, \dot{Y})-\beta_{e} \dot{Y}\right]^{2}\right\}=\min
$$

Which also equals to equation(4), so the formally identical criterion (4) is used in both stochastic linearization and partial linearization, the ensemble averaging in equation (4) is performed with different probability densities for these two methods. In the stochastic linearization, the probability density is assumed to be Gaussian, while in the present partial linearization method, it is generally not Gaussian(Elishakoff and Cai, 1993).

In equation(1), the left side is calculated. Ensemble averaging of equation result in

$\frac{d}{d t} E\left[Y_{2}^{2}\right]=-2 E\left[Y_{2}\left[f\left(Y_{1}, Y_{2}\right)+g\left(Y_{1}\right)\right]\right]+2 \pi K$

Which reduces to

$$
E\left[Y_{2} f\left(Y_{1}, Y_{2}\right)\right]=\pi K
$$

Here, a remarkable conclusion is leaded: the average work done by the damping force per unit time depends only on the spectral density of the excitation, regardless of the damping mechanism, for an oscillator with only an additive white noise excitation. Which is consistent with Karnopp's conclusion by using a different proof(Karnopp, 1967). According to equation (3) and (7),

$$
\beta_{e}=\frac{E[\dot{Y} \cdot f(Y, \dot{Y})]}{E\left[\dot{Y}^{2}\right]}=\frac{\pi K}{E\left[\dot{Y}^{2}\right]}
$$

The exact stationary probabilistic solution of equation (2) is a classical result of the random vibration,

$$
p(y, \dot{y})=C \exp \left[-\frac{\beta_{e}}{\pi K}\left(\frac{\dot{y}^{2}}{2}+\int_{0}^{y} g(u) d u\right)\right]
$$

Where $\mathrm{C}$ is a constant determined from the normalization condition

$$
C=\int_{-\infty}^{+\infty} \int_{-\infty}^{+\infty} \exp \left[-\frac{\beta_{e}}{\pi K}\left(\frac{\dot{y}^{2}}{2}+\int_{0}^{y} g(u) d u\right)\right] d y d \dot{y}
$$

The probability density (9) can be considered as an approximate one for the response of the original system and can be used in equation (7)to yield 
$C \int_{-\infty}^{+\infty} \int_{-\infty}^{+\infty}\left\{\left[\begin{array}{l}\dot{y} f(y, \dot{y}) \exp \\ \left.\left[-\frac{\beta_{e}}{\pi K}\left(\frac{\dot{y}^{2}}{2}+\int_{0}^{y} g(u) d u\right)\right]\right\} d y d \dot{y}=\pi K\end{array}\right.\right.$

Substituting equation(10) into equation(11), we obtain

$$
\int_{-\infty}^{+\infty} \int_{-\infty}^{+\infty}\left\{\left[-\frac{\beta_{e}}{\pi K}\left(\frac{\dot{y}^{2}}{2}+\int_{0}^{y} g(u) d u\right)\right]\right\} d y d \dot{y}=0
$$

\section{Certification of equivalent damping coefficient for test ship}

Suppose the rolling motions are uncoupled and the added mass term is independent of frequency. According to the typical single-degree- ${ }^{-}$-f - freedom (SDF) differential equation of intact ship rolling considering the nonlinearity in restoring moment and damping, the final form of the differential equation of motion is obtained as follows(Long, et al., 2009):

$$
\ddot{\phi}+d_{1} \dot{\phi}+d_{3} \dot{\phi}^{3}+k_{1} \phi+k_{3} \phi^{3}=m(t)
$$

Where

$$
\begin{array}{ll}
d_{i}=D_{i} /\left(I_{44}+A_{44}\right) & i=1,3 \\
k_{j}=K_{j} /\left(I_{44}+A_{44}\right) & j=1,3 \\
m(t)=M(t) /\left(I_{44}+A_{44}\right) &
\end{array}
$$

The equation is known as the hardening type Duffing oscillator when $\mathrm{k} 3$ is positive and the softening type Duffing oscillator when k3 is negative. Apply the partial linearization method, the above equation can be written as follows.

$$
\ddot{\phi}+\beta_{e} \dot{\phi}+k_{1} \phi+k_{3} \phi^{3}=m(t)
$$

Where

$$
\beta_{e}=\frac{d_{1}}{2}+\left[\left(\frac{d_{1}}{2}\right)^{2}+3 d_{3} \pi K\right]^{1 / 2}
$$

From above equation, once $\beta e$ is determined, the approximate joint probability density (9) is also determined. In Fig. 1, the stationary mean square values of the displacement $\mathrm{X}$ for sytem are plotted against the stiffness nonlinearity parameter and he damping nonlinearity parameter respectively. Results computed from both the 'full' linearization and partial linearization are shown and compared with the Monte Carlo simulation results(Cai, 2004). The higher accuracy can be achieved with the present method as compared to the "full" linearization
procedure(Elishakoff and Cai, 1993). It is shown that the partial linearization method is a consistent approximation scheme in the sense that the obtained approximate probability density for certain statistical moments of the system response.

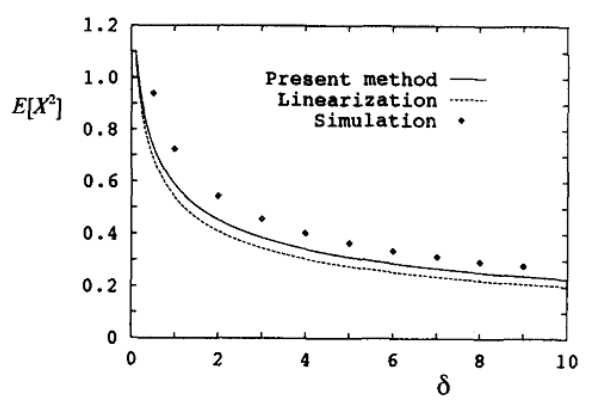

Fig. 1 Mean square displacement with respect to stiffness nonlinearity: $\mathrm{K}=1, \mathrm{~d} 1=0.1, \mathrm{~d} 3=0.5, \mathrm{k} 1=1, \mathrm{k} 3=0$ (Elishakoff and Cai, 1993)

\section{Results and discussions}

After the coefficient $\beta e$ are certified, the rolling oscillation equation of the ferry sails in beam seas can be described by adding the nonlinear term to the righting lever in the following form(Ikeda, et al., 2008, Lee, et al., 2009)

$$
\ddot{\phi}+2 v \dot{\phi}+\omega_{0}^{2}\left(\phi+k \phi^{3}\right)=\omega_{0}^{2} \Theta \sin \omega_{e} t
$$

Where

$$
\begin{aligned}
& k=\frac{k_{3}}{\omega_{0}^{2}} \\
& m(t)=\omega_{0}^{2} \Theta \sin \omega_{e} t
\end{aligned}
$$

When the ferry is in calm water, an impulsive disturbance in roll or roll velocity, such as that caused by a wind gust, can set up an oscillatory roll motion. The period of such roll motion in calm water depends on the ship's stability or restoring moment properties, damping properties and the mass properties(Jiang, et al., 2000, Shin, et al., 2004). A sample rolling oscillation of such a ferry is shown in Fig. 4

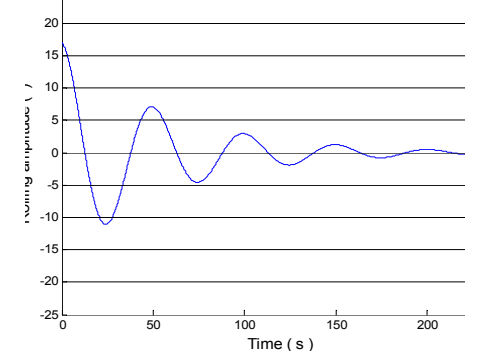

Fig. 4 A sample rolling decay in still water 
Actually, it is difficult to obtain the exact solution of the rolling motion(Davies and Liu, 1990, Jung-mo, et al., 2008), Here, an approximate solution is obtained by the following method. The initial approximation of the equation is chosen as follows:

$$
\phi=A \sin \left(\omega_{e} t-\varepsilon\right)
$$

Then

$$
\begin{aligned}
& \dot{\phi}=A \omega_{e} \cos \left(\omega_{e} t-\varepsilon\right) \\
& \ddot{\phi}=-A \omega_{e}^{2} \sin \left(\omega_{e} t-\varepsilon\right)
\end{aligned}
$$

These equation leads to the following two formulae:

$$
\begin{aligned}
& \dot{A}=\dot{\phi}\left(\frac{\phi}{A}+\frac{\ddot{\phi}}{A \omega^{2}}\right) \\
& \dot{\varepsilon}=\omega-\frac{\dot{\phi}^{2}-\ddot{\phi} \phi}{\omega A^{2}}
\end{aligned}
$$

Because the derivatives changes with time, so the equations can be written as follows,

$$
\begin{aligned}
& \dot{A}=\frac{1}{T} \int_{0}^{T} \dot{\phi}\left(\frac{\phi}{A}+\frac{\ddot{\phi}}{A \omega^{2}}\right) d t \\
& \dot{\varepsilon}=\frac{1}{T} \int_{0}^{T}\left(\omega-\frac{\dot{\phi}^{2}-\ddot{\phi} \phi}{\omega A^{2}}\right) d t
\end{aligned}
$$

Substitute equation (20) in to above, we can obtain the average derivatives as follows

$$
\begin{aligned}
& \dot{A}=-\alpha A+\omega\left(\frac{\omega_{0}}{\omega}\right)^{2} \frac{\Theta \sin \varepsilon}{2} \\
& \dot{\varepsilon}=\frac{\omega}{2}\left[\begin{array}{l}
1-\left(\frac{\omega_{0}}{\omega}\right)^{2}-\left(\frac{\omega_{0}}{\omega}\right)^{2} \frac{3 \kappa A^{2}}{4} \\
+\left(\frac{\omega_{0}}{\omega}\right)^{2} \frac{\Theta \cos \varepsilon}{A}
\end{array}\right]
\end{aligned}
$$

Finally, we can obtain the relationship between the $\omega / \omega 0$ and roll amplitude $\mathrm{A}$.

$$
\begin{aligned}
& \frac{\omega}{\omega_{0}}=\left[\xi-\psi \pm\left(\zeta^{2}+\psi^{2}-2 \xi \psi\right)^{1 / 2}\right]^{1 / 2} \\
& \xi=1+\frac{3 \kappa \phi_{a}^{2}}{4}, \quad \psi=2\left(\frac{N \phi_{a}}{\pi}\right)^{2}, \quad \zeta=\frac{\Theta}{A}
\end{aligned}
$$

Then, the effects of variation in wave slope and damping parameters on the response of the oscillation system can be resolved. Fig. 2 shows the synchronous resonance curves of rolling oscillation for partial linearization damping with nonlinear restoring moment. The curves demonstrate that the effect of the variation in wave slope on the amplitude of the relative rolling oscillation. The rolling peak amplitude increase as the wave slope increases.
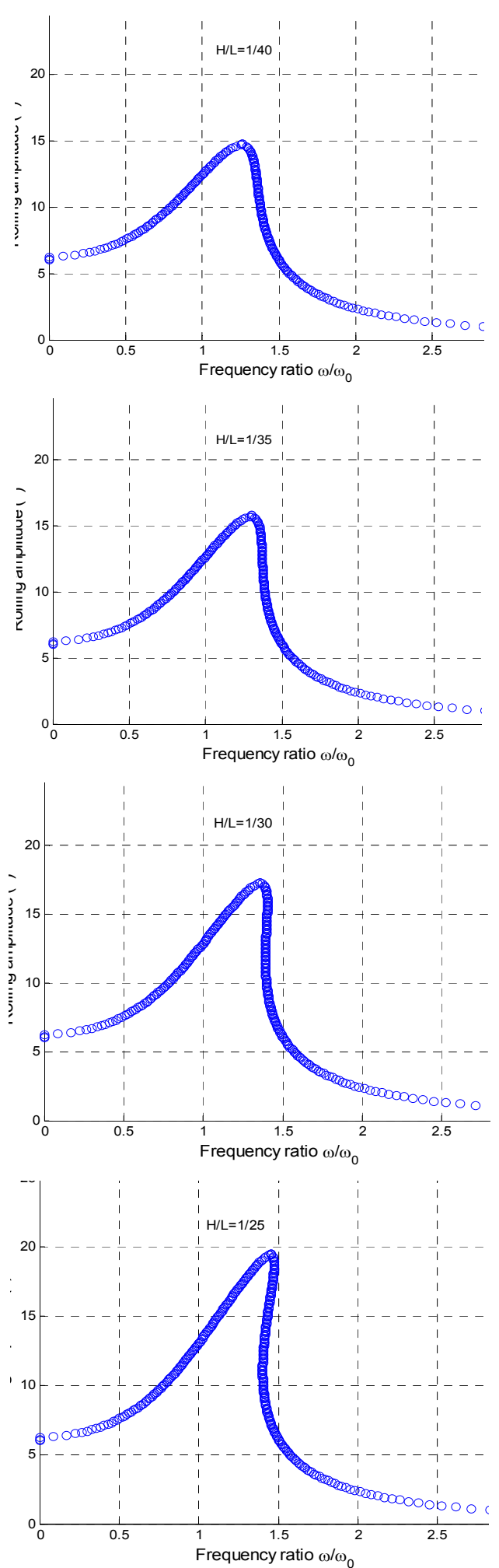

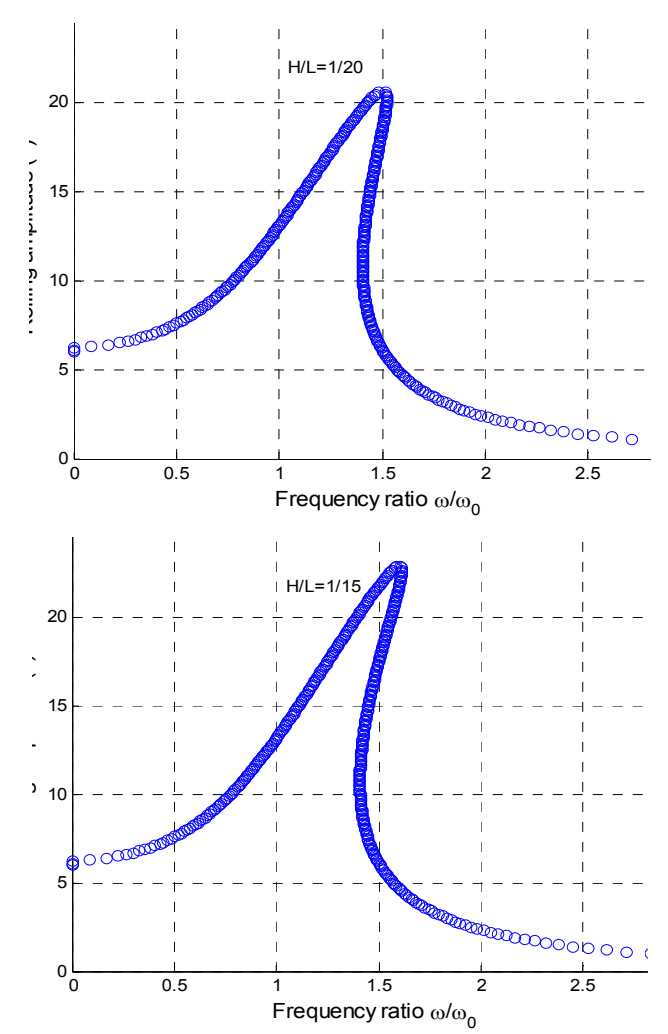

Fig. 2 Roll response for partial stochastic linearization damping with nonlinear restoring moment

\section{Conclusions}

This research studied the capsizing condition of a ferry considering hydrodynamic parameters in beam seas in addition to the characteristics of rolling damping and restoring moment. The linearity simplification equation for the single-degree-of-freedom nonlinear rolling oscillator, which subjected to an external variable excitation, has worked out based on the partial linearization method, which gives more accurate results than standard equivalent linearization method and best approximates the true system in some sense. Before the end of the study, the approximating solution to the stationary nonlinear random rolling oscillation problems was resolved.

The work focuses on the importance of damping coefficient and variation of the wave slope on the roll response of a vessel(Lin and Yim, 1995). The calculation results demonstrate the complexity of nonlinear resonance of rolling oscillation. The effects of damping and wave steepness are not neglect for vessel design and analysis. As not much work has been done here, it is hoped that the paper will provide a preliminary sketch and will be useful for the designers to arrive at a proper reserve margin for the stability in resonant conditions considering waves and other environmental conditions. Future work includes testing the method on multiple degree of freedom systems, extending the method to non-stationary excitation and investigating what weighting functions should be used in the norms.

\section{Acknowledgements}

This work was supported by Underwater Vehicle Research Center (UVRC) and Agency for Defense Development (ADD) of Korea.

\section{References}

[1] Belenky, V. L., Degtyarev, A. B., and Boukhanovsky, A. V. (1998), "Probabilistic qualities of nonlinear stochastic rolling," Ocean Engineering, Vol. 25, No. 1, pp. 1-25.

[2] Birman, V. (1986), "On the nonlinear uncoupled roll and pitch of submerged vehicles," Ocean Engineering, Vol. 13, No. 6, pp. 621-625.

[3] Brukner, A. and Lin, Y. K. (1987), "Generalization of the equivalent linearization method for non-linear random vibration problems," International Journal of Non-Linear Mechanics, Vol. 22, No. 3, pp. 227-235.

[4] Cai, G. Q. (2004), "Non-linear systems of multiple degrees of freedom under both additive and multiplicative random excitations," Journal of Sound and Vibration, Vol. 278, No. 4-5, pp. 889-901.

[5] Cai, G. Q. and Lin, Y. K. (1988), "On exact stationary solutions of equivalent non-linear stochastic systems," International Journal of Non-Linear Mechanics, Vol. 23, No. 4, pp. 315-325.

[6] Caughey, T. K. (1986), "On the response of non-linear oscillators to stochastic excitation," Probabilistic Engineering Mechanics, Vol. 1, No. 1, pp. 2-4.

[7] Chakrabarti, S. (2001), "Empirical calculation of roll damping for ships and barges," Ocean Engineering, Vol. 28, No. 7, pp. 915-932.

[8] Contento, G., Francescutto, A., and Piciullo, M. (1996), "On the effectiveness of constant coefficients roll motion equation," Ocean Engineering, Vol. 23, No. 7, pp. 597-618.

[9] Dalzell, J. F. (1978), "A note on the form of ship roll damping," Journal of Ship Research, Vol. 22, No. 3, pp. $178-185$.

[10] Davies, H. G. and Liu, Q. (1990), "The response envelope probability density function of a Duffing oscillator with random narrow-band excitation," Journal of Sound and Vibration, Vol. 139, No. 1, pp. 1-8. 
[11] Elishakoff, I. and Cai, G. Q. (1993), "Approximate solution for nonlinear random vibration problems by partial stochastic linearization," Probabilistic Engineering Mechanics, Vol. 8, No. 3-4, pp. 233-237.

[12] Epele, L. N., Fanchiotti, H., and Spina, A. (1985), "Path-integral approach to nonlinear self-excited oscillators," Physical Review A, Vol. 31, No. 1, pp. 463.

[13] Haddara, M. R. and Zhang, Y. (1994), "On the Joint Probability Density Function of Non-Linear Rolling Motion," Journal of Sound and Vibration, Vol. 169, No. 4, pp. 562-569.

[14] Ikeda, Y., Yamamoto, N., and Fukunaga, K. (2008), "Seakeeping Performances of a Large Waves-Piercing Catamaran in Beam Waves," presented at the The 6th Osaka Colloquium on Seakeeping and Stability of Ships.

[15] Jiang, C., Troesch, A. W., and Shaw, S. W. (2000), "Capsize criteria for ship models with memory-dependent hydrodynamics and random excitation," Philosophical Transactions of the Royal Society of London. Series A: Mathematical, Physical and Engineering Sciences, Vol. 358, No. 1771, pp. 1761-1791.

[16] Jung-mo, Y., Tabeta, S., and Sato, T. (2008), "respect to the acceptability of ship grounding risks," in OCEANS 2008 - MTS/IEEE Kobe Techno-Ocean, pp. 1-7.

[17] Karnopp, D. (1967), "Power balance method for nonlinear random vibration," Journal of Applied Mechanics, Vol. 34, No. 1, pp. 212-14.

[18] Langley, R. S. (1988), "An investigation of multiple solutions yielded by the equivalent linearization method," Journal of Sound and Vibration, Vol. 127, No. 2, pp. 271-281.

[19] Lee, D., Hong, S. Y., and Lee, G. J. (2007), "Theoretical and experimental study on dynamic behavior of a damaged ship in waves," Ocean Engineering, Vol. 34, No. 1, pp. 21-31.

[20] Lee, S. K., Long, Z. J., and Jeong, J. H. (2009), "Risk assessment method of simulation-based for the intact ship stability," presented at the International Conference on Computational \& Experimental Engineering and Sciences(ICCES'09), Phuket, Thailand.

[21] Lee, S. K. (2000), "Stability Analysis of an Initially Inclined Ship in Following Sea," Journal of Applied Mechanics, Vol. 67, No. 4, pp. 717-719.

[22] Lin, H. and Yim, S. C. S. (1995), "Chaotic roll motion and capsize of ships under periodic excitation with random noise," Applied Ocean Research, Vol. 17, No. 3, pp. 185-204.

[23] Long, Z. J., Lee, S. K., and Lee, S. J. (2009),
"Probabilstic Prediction of Stability of Ship by Risk Based Approach," International Journal of Navigation And Port Research, Vol. 33, No. 4, pp. 255-261.

[24] Ochi, M. K. (1986), "Non-Gaussian random processes in ocean engineering," Probabilistic Engineering Mechanics, Vol. 1, No. 1, pp. 28-39.

[25] Polidori, D. C. and Beck, J. L. (1996), "Approximate solutions for non-linear random vibration problems," Probabilistic Engineering Mechanics, Vol. 11, No. 3, pp. 179-185.

[26] Proppe, C., Pradlwarter, H. J., and Schuler, G. I. (2003), "Equivalent linearization and Monte Carlo simulation in stochastic dynamics," Probabilistic Engineering Mechanics, Vol. 18, No. 1, pp. 1-15.

[27] Shin, Y. S., Belenky, V. L., and Paulling, J. R. (2004), "Criteria for parametric roll of large containerships in longitudinal seas," Transactions Society of Naval Architects and Marine Engineers, Vol. 112, pp. 14-47.

[28] Socha, L. (2005), "Linearization in Analysis of Nonlinear Stochastic Systems: Recent Results ${ }^{--}$Part I: Theory," Applied Mechanics Reviews, Vol. 58, No. 3, pp. 178-205.

[29] Surendran, S., Lee, S. K., and Reddy, J. V. R. (2005), "Non-linear roll dynamics of a Ro-Ro ship in waves," Ocean Engineering, Vol. 32, No. 14-15, pp. 1818-1828.

[30] Surendran, S., Lee, S. K., and Sohn, K. H. (2007), "Simplified model for predicting the onset of parametric rolling," Ocean Engineering, Vol. 34, No. 3-4, pp. 630-637.

[31] Surendran, S. and Venkata Ramana Reddy, J. (2003), "Numerical simulation of ship stability for dynamic environment," Ocean Engineering, Vol. 30, No. 10, pp. 1305-1317.

[32] Zhu, W. Q., Soong, T. T., and Lei, Y. (1994), "Equivalent nonlinear system method for stochastically excited Hamiltonian systems," Journal of applied mechanics, Vol. 61, No. 3, pp. 618-623.

Received 1 October 2009.

Revised 28 October 2009.

Accepted 28 October 2009. 